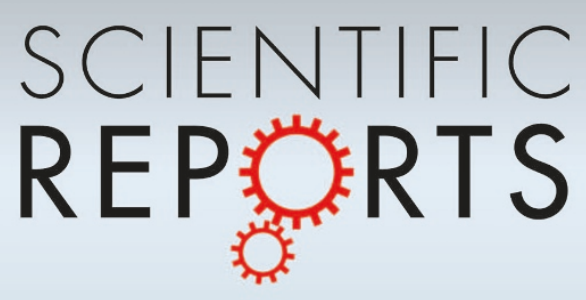

OPEN

SUBJECT AREAS:

PCR-BASED TECHNIQUES

HIGH-THROUGHPUT SCREENING

Received

3 September 2014

Accepted

3 December 2014

Published

5 January 2015

Correspondence and requests for materials should be addressed to F.W.L. (lifeiwu3394@ gmail.com) or G.W. (wugang@caas.cn)

* These authors contributed equally to this work.

\section{Development and Validation of $A$ 48-Target Analytical Method for High-throughput Monitoring of Genetically Modified Organisms}

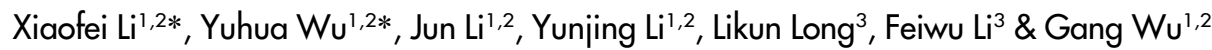

\begin{abstract}
'Key Laboratory of Oil Crop Biology, Ministry of Agriculture, Oil Crops Research Institute, Chinese Academy of Agricultural Sciences, No. 2 Xudong 2nd Road, Wuhan 430062, People's Republic of China, ${ }^{2}$ Supervision and Test Center (Wuhan) for Environmental Safety of Genetically Modified Plants, Ministry of Agriculture, No. 2 Xudong 2nd Road, Wuhan 430062, People's Republic of China, ${ }^{3}$ Agro-Biotechnology Research Institute, Jilin Academy of Agricultural Sciences, No. 1363 Caiyu Avenue, Changchun 130033, People's Republic of China.
\end{abstract}

The rapid increase in the number of genetically modified (GM) varieties has led to a demand for high-throughput methods to detect genetically modified organisms (GMOs). We describe a new dynamic array-based high throughput method to simultaneously detect 48 targets in 48 samples on a Fludigm system. The test targets included species-specific genes, common screening elements, most of the Chinese-approved GM events, and several unapproved events. The 48 TaqMan assays successfully amplified products from both single-event samples and complex samples with a GMO DNA amount of $0.05 \mathrm{ng}$, and displayed high specificity. To improve the sensitivity of detection, a preamplification step for 48 pooled targets was added to enrich the amount of template before performing dynamic chip assays. This dynamic chip-based method allowed the synchronous high-throughput detection of multiple targets in multiple samples. Thus, it represents an efficient, qualitative method for GMO multi-detection.

W ith the rapid development of biotechnology, the production of genetically modified (GM) crops has increased continuously since the commercial planting of the first approved GM variety in 1996. According to the International Service for the Acquisition of Agri-Biotech Applications (ISAAA), 27 types of plants and 336 transgenic varieties had been developed and commercialized worldwide by the end of 2013, and the planting area of GM crops was greater than 175 million hectares in 27 countries $^{1}$. Many new GM varieties are entering field trials or nearing environmental release. In many countries, the government and the public have a degree of distrust for genetically modified organisms (GMOs) because of perceived risks to food safety and the environment ${ }^{2}$. To protect the consumer's right to information, many countries have developed legislation to regulate and track the presence of GMOs in feed and foodstuffs $\mathrm{s}^{3,4}$.

To comply with GMO legislation and to ensure product legality and traceability, effective and accurate analytical methods are essential for GMO screening, identification, and quantification. The DNA-based polymerase chain reaction (PCR) technique is the most widely accepted GMO detection method, and has been classified into screening, gene-specific, construct-specific, and transgenic event-specific methods according to the level of specificity ${ }^{3,5}$. During GMO detection, a series of PCRs are performed sequentially to determine the identity of GMO ingredients (authorized or unauthorized) in the test samples, first screening for elements generally used in GMOs, and then for event-specific sequences. Because of the large number and complexity of GM varieties, it is generally not feasible to conduct traditional single-target PCRs for all possible elements and events. Also, the traditional detection strategy is time-consuming, laborious, and costly ${ }^{6}$. Because of the rapid increase in the number of new GMOs, there is a need for high-throughput screening and identification methods to simultaneously detect multiple targets, in multiple samples, in a single experiment. Many multiple-target detection methods for GMO screening and identification have been reported, including multiplex PCR, multiplex PCR-based techniques, and the ready-to-use real time PCR matrix $x^{3,7-10}$.

The objective of multiplex PCR is to detect multiple targets simultaneously in a single reaction. Multiplex PCR often requires careful optimization of the composition and concentration of reagents and primers ${ }^{7,11}$. 
Conventional multiplex PCR can detect only five to six targets because of the poor separation of the PCR products in ordinary electrophoresis ${ }^{8}$. The multiplexing capability of multiplex real-time PCR is restricted by the number of channels in the real-time PCR thermal cycler, and by the number of available fluorescent dyes ${ }^{12-14}$. To improve the multiplexing capability in GMO detection, new detection strategies have been introduced and applied to GMO diagnostics. Such strategies combine multiplex PCR with capillary gel electrophoresis (CGE) or microarray hybridization. In particular, DNA microarray assays have shown considerable potential for the simultaneous analysis of thousands of different targets ${ }^{10,15-19}$. However, in a practical context, the target throughput for both the multiplex PCR-based CGE method and the microarray method is often limited by the multiplicities of multiplex $\mathrm{PCR}^{15,20,21}$. Other limitations of multiplex PCR include the preferential amplification of partial targets, and non-specific background amplification resulting from interference of multiple primer combinations in a single reaction ${ }^{22,23}$. To overcome the defects of multiplex PCR associated with end-point PCR technology, an initial multiplex preamplification step with a low concentration of initial primers and a low PCR amplification cycle number have been used to increase the amount of template. This strategy has been used in several high-throughput DNA detection methods, including nucleic acid sequencebased amplification (NASBA) implemented microarray analysis (NAIMA), multiplex quantitative DNA array-based PCR (MQDAPCR), and multiplex microdroplet PCR implemented capillary gel electrophoresis (MPIC) ${ }^{9,20,24}$. A recently reported high-throughput method, MACRO, consists of multiplex amplification on a chip, and uses an oligo microarray for readouts of multiple amplicons. This method simultaneously detected 91 targets $^{25}$. In general, microarraybased analytical approaches are very complex and expensive, and are therefore impractical for GMO detection.

The ready-to-use real-time PCR matrix is a 96-well or 384-well prespotted plate containing lyophilized primers and probes, allowing the simultaneous detection of multiple targets ${ }^{26,27}$. The real-time PCR-based ready-to-use 96-well plate was developed by the Joint Research Centre to analyze two samples per plate, detecting 48 individual targets ${ }^{26}$. Then, a multiplex real-time PCR method was established to simultaneously perform 24 multiplex real-time PCRs on a 384-well ready-to-use plate, allowing the detection of 47 targets in seven samples in duplicate ${ }^{27}$. Compared with multiplex PCR, the use of prespotted plates is more flexible, and testing parameters can be added or removed as needed without the need to re-optimize the entire system. However, the ready-to-use multi-target analytical system can analyze only a few samples in a single experiment because of the trade-off between the target throughput and sample throughput.

The Fluidigm system with an integrated fluidic circuit (IFC) chip is a new high-throughput detection platform. The IFC chip used in the Fluidigm system includes digital and dynamic arrays. The digital arrays allow the precise quantification of nucleic acids. This system has been used to measure variations in copy number and to perform molecular diagnostics of lung cancer, gastrointestinal cancer, and other diseases ${ }^{28-31}$. In GMO analysis, digital array PCR has been used to assess detection limits ${ }^{32}$. Microfluidic dynamic array technology has been used in a number of high-throughput analyses such as gene expression analysis, microRNA expression analysis, and single cell gene expression analysis ${ }^{33-36}$. Currently, the IFC chips available for dynamic analysis include the 48.48 (48 samples*48 assays), the 96.96 (96 samples*96 assays), and the 192.24 (192 samples*24 assays). Compared with other methods, the dynamic chip method can maintain compatibility by using validated PCR protocols in a highthroughput format. In 2014, Brod et al. first reported a high throughput method targeting 28 GMO elements using dynamic chips ${ }^{37}$.

Since 1994, 62 countries and regions ( 35 countries +27 member states of the EU) have granted regulatory approval for 336 transgenic events for food and/or feed use and for environmental release or planting. The type and number of approved events differs among different countries ${ }^{1}$. At present, 37 kinds of GM crops have been approved by China for import as raw materials for processing. During GMO detection, the more parameters are tested, the higher the chance of detecting a GMO ingredient. Therefore, we developed a 48-plex higher-throughput GMO detection method based on validated real-time PCR methods and the dynamic IFC chip. This method allowed the simultaneous high-throughput detection of multiple targets in multiple samples. The targets included speciesspecific genes, regulatory elements, target genes, most of the Chinese approved transgenic events, and several unapproved events.

\section{Results}

Selection of targets. We selected detection targets based on the current Chinese GMO regulations. The detection targets included seven screening elements (P-CaMV35S, P-FMV35S, T-NOS, NPTII, Cry1Ab, Bar, and Pat), 36 transgenic events (transgenic cotton MON531, MON88913, MON1445, MON15985, LLCotton25, GHB614; transgenic maize 3272, 59122, Bt176, Bt11, GA21, MIR162, MIR604, MON810, MON863, MON88017, MON89034, NK603, T25, TC1507; transgenic rapeseed MS1, Topas 19/2, OXY235, MS8, RF3, RT73, T45; transgenic soybean 40-3-2, A2704-12, MON89788, MON87701, DP-356043, A5547-127, CV-127, DP305423-1; transgenic rice TT51-1) and 5 reference genes (cotton, maize, rapeseed, rice, and soybean). The selected targets covered either $100 \%(37 / 37)$ approved transgenic varieties or three unapproved events TT51-1, MIR162, and A5547-127 in China. Out of the 37 Chinese approved GM varieties, 31 could be detected based on the above seven screening elements; the six events that could not be detected based on these elements were GM soybean MON89788, MON87701, DP356043, DP305423, BPS-CV127-9 and GM cotton GHB614. These lines do not harbour commonly used screening targets, and so they must be analyzed using event-specific methods.

Data on the presence of regulatory elements and genes in GM crops in the GM crops database indicate that these 43 foreign DNA targets could be used to detect most of the released transgenic events, including 100\% (16/16) of transgenic rapeseed, 96.4\% (27/28) of transgenic maize, $92.3 \%(13 / 14)$ of transgenic cotton, $100 \%(2 / 2)$ of transgenic rice, $84.6 \%$ (11/13) of transgenic soybean, and $93 \%$ (28/ $30)$ of other transgenic crops. Therefore, the targets selected in this study not only allow detection and identification of Chinese authorized GM crops, but also allow the detection of unauthorized transgenic events in test samples.

Validation of real-time PCR methods. We selected 36 eventspecific methods to establish the high-throughput system. The MON87701 soybean was not available, and so the specificity and sensitivity of the MON87701-specfic method could not be validated. To test the specificity of the other 35 event-specific methods, two samples were prepared for each TaqMan assay. One was the DNA solution from the corresponding transgenic event; and the other was a mixture of 34 events, that is, all events except for the corresponding event. Real-time PCR reactions were run with these samples as the template for each TaqMan assay, in triplicate. The amplification results showed that these 35 event-specific methods identified specific transgenic events, and no typical amplification plots were produced from mixed samples. The results of the specificity test also indicated that there was no cross-contamination among different events during genomic DNA extraction. The specificity of seven screening methods was also validated by amplifying the corresponding targets in 35 transgenic events, displaying expected specificity.

To test the sensitivity of the detection methods, the genomic DNA was diluted to 10 and 5 copies $/ \mu \mathrm{L}$. Ten copies of target DNA could be consistently detected for all of the real-time PCR methods. These 
results suggested that the primer/probe sets used in this study had high specificity and good sensitivity, and were suitable for use in subsequent experiments.

Preamplification. Only a few reaction chambers produced a fluorescence signal when the 35 single-event DNA solutions at $0.5 \mathrm{ng} / \mu \mathrm{L}$ level and 11 complex samples (S36-S46) were directly used as DNA templates to run dynamic chip assays on the Biomark system (Fig. 1). Based on the genome size of the five crop species $^{38}$, the template concentration, and the chamber volume $(9 \mathrm{~nL})$ of the 48.48 dynamic chips, the copy number of haploid genomes per reaction chamber was determined to be 2.2 for rice, 0.3 for maize, 0.4 for cotton, and 1.0 for rapeseed and soybean. These results indicated that the amount of template was too low to generate an amplification plot. Therefore, the sensitivity of more than $0.5 \mathrm{ng}$ GM DNA for the dynamic chip was insufficient for real GMO samples.

To increase the sensitivity of the dynamic chip system, preamplification reactions were performed with a PreAmp Master Mix kit to increase the amount of template. An average of three PCR templates per well is the minimum amount to detect the presence of the target at the $95 \%$ confidence level, and for the samples of $0.1 \%$ level the copy number of the reference gene target needs to be amplified to at least
3000. Therefore, the preamplification would require at least 8-9 cycles to meet the requirements for sensitivity. Considering PCR efficiency is usually less than $100 \%$ in practice, a 14 -cycle preamplification was performed, as recommended by the manufacturer.

To check the preamplification results, using $0.5 \mathrm{ng}$ of GM DNA and preamplification products from eight transgenic events in five crops as templates, partial TaqMan assays were carried out to compare the $\mathrm{Ct}$ values of templates before and after preamplification (Table 1). The Ct values of detection targets after preamplification were significantly decreased by an average of 10.1, compared with those before preamplification (Table 1). This result indicated that the abundance of targets in the template was greatly increased. However, the delta Ct values between the original template and preamplified template differed among the targets (Table 1). This result suggested that the ratio of different targets after preamplification deviated from that before preamplification, and the preamplification products were not suitable for quantitative analysis of GMO content in original samples. Consequently, we used preamplified products only for qualitative detection in subsequent analyses.

Sensitivity of dynamic chip assays. The genomic DNA from transgenic rapeseed Topas 19/2 and OXY-235 were serially diluted to $0.5,0.05,0.025$ and $0.005 \mathrm{ng} / \mu \mathrm{L}$ using salmon sperm DNA. The

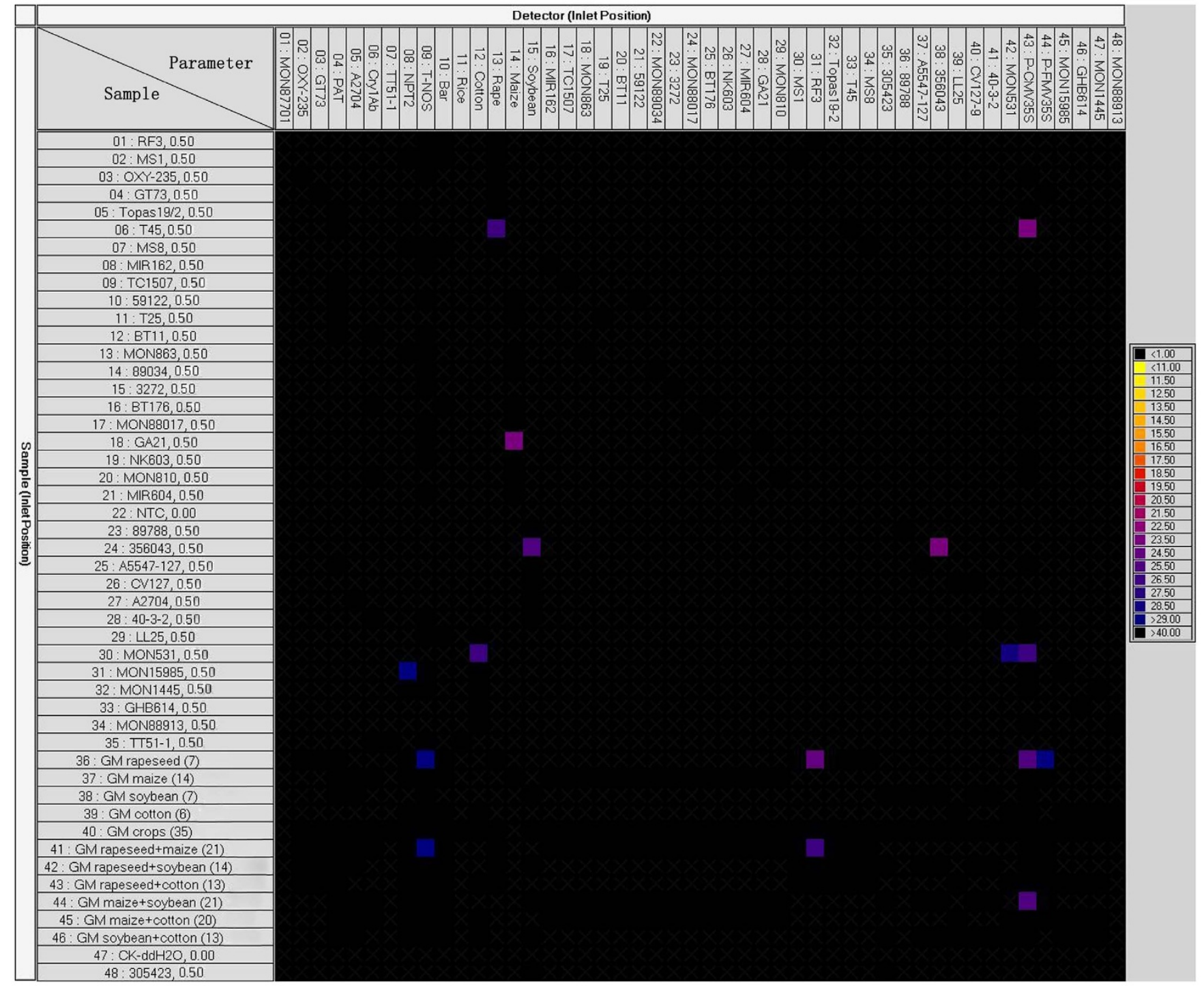

Figure 1 | Panel readouts from the dynamic chip using $0.5 \mathrm{ng}$ GM DNA solutions from test samples as templates. Coloured squares correspond to positive hits on the chip, black squares indicate chambers with no amplification. 
Table 1 | Test of preamplification efficiency by TaqMan assays

\begin{tabular}{|c|c|c|c|c|c|}
\hline Event & TaqMan assays & $\begin{array}{c}\mathrm{C}+\text { Value } \\
\text { After pre-amplification }\end{array}$ & $\begin{array}{c}\mathrm{C} t \text { Value } \\
\text { Before preamplification }\end{array}$ & $\Delta \mathrm{Ct}$ & Mean \\
\hline BT176 & Bar & 20.01 & 30.32 & 10.31 & 10.10 \\
\hline MON863 & NPTII & 22.87 & 32.41 & 9.54 & \\
\hline NK603 & NK603 & 24.15 & 34.45 & 10.3 & \\
\hline GTS40-3-2 & P-CaMV35S & 20.84 & 31.54 & 10.7 & \\
\hline ŁL25 & T-NOS & 23.26 & 33.59 & 10.33 & \\
\hline T45 & PAT & 23.78 & 34.16 & 10.38 & \\
\hline OXY-235 & OXY-235 & 25.74 & 35.31 & 9.57 & \\
\hline$\pi 51-1$ & $\pi 51-1$ & 24.16 & 34.32 & 10.16 & \\
\hline ПТ51-1 & CrylAb & 22.21 & 31.86 & 9.65 & \\
\hline
\end{tabular}

diluted DNA samples were used as templates in the preamplification reactions with pooled TaqMan assay mixture. After preamplification, the diluted preamplification products were used in real-time PCR analyses on dynamic chips to test the sensitivity of the dynamic chip assays. In this run, there were six replicates of seven samples, five replicates of $0.005 \mathrm{ng}$ Topas 19/2, and one NTC control (No 22 sample). Eight targets including the rapeseed reference gene, NPTII, CaMV35S, FMV35S, T-NOS, PAT, Topas 19/2, and OXY-235, were assayed with six parallels per target (Fig. 2). As shown in Fig. 2, the NTC produced no amplification signal, and $0.5 \mathrm{ng}$ DNA of both

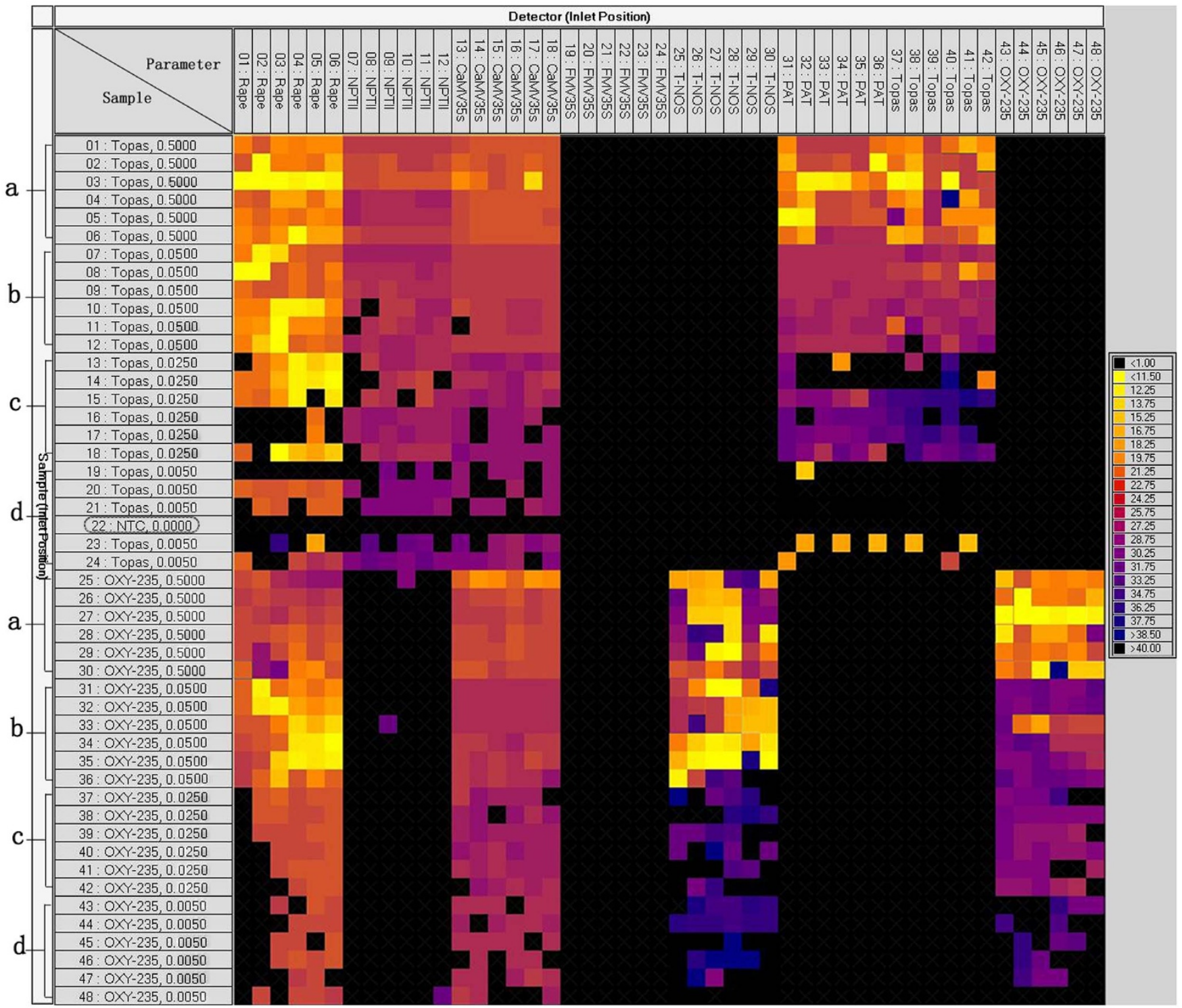

Figure $2 \mid$ Analytical sensitivity of dynamic chip assays in detecting serially diluted genomic DNA solutions from Topas 19/2 and OXY-235 events. a-d correspond to GMO DNA amount of $0.5 \mathrm{ng}, 0.05 \mathrm{ng}, 0.025 \mathrm{ng}$, and $0.005 \mathrm{ng}$, respectively. Coloured chambers indicate positive signals; black chambers indicate negative signals. 


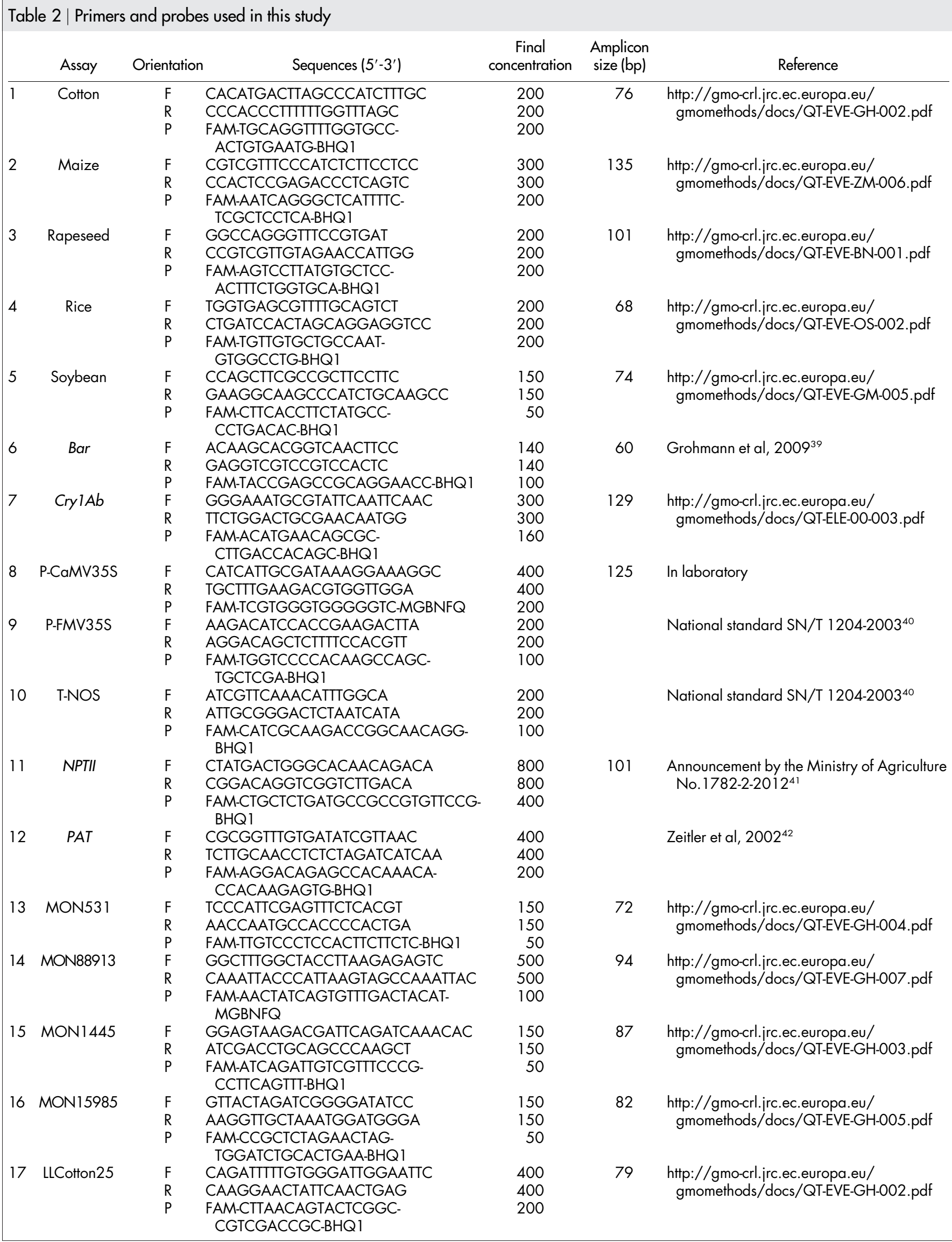


Table 2 | Continued

\begin{tabular}{|c|c|c|c|c|c|c|}
\hline & Assay & Orientation & Sequences $\left(5^{\prime}-3^{\prime}\right)$ & $\begin{array}{c}\text { Final } \\
\text { concentration }\end{array}$ & $\begin{array}{l}\text { Amplicon } \\
\text { size (bp) }\end{array}$ & Reference \\
\hline 18 & GHB614 & $\begin{array}{l}F \\
R \\
P\end{array}$ & $\begin{array}{l}\text { CAAATACACTTGGAACGACTTCGT } \\
\text { GCAGGCATGCAAGCTTTAAAA } \\
\text { FAM-CTCCATGGCGATCGCT- } \\
\text { ACGTTCTAGAATT-BHQ1 }\end{array}$ & $\begin{array}{l}400 \\
400 \\
200\end{array}$ & 120 & $\begin{array}{l}\text { http://gmo-crl.jrc.ec.europa.eu/ } \\
\text { gmomethods/docs/QT-EVE-GH-006.pdf }\end{array}$ \\
\hline 19 & 3272 & $\begin{array}{l}F \\
R \\
P\end{array}$ & $\begin{array}{l}\text { TCATCAGACCAGATTCTCTTTTATGG } \\
\text { CGTTCCCGCCTTCAGTTTA } \\
\text { FAM-ACTGCTGACGCGG- } \\
\text { CCAAACACTG-BHQI }\end{array}$ & $\begin{array}{r}50 \\
900 \\
200\end{array}$ & 95 & $\begin{array}{l}\text { hitp://gmo-crl.jrc.ec.europa.eu/ } \\
\text { gmomethods/docs/QT-EVE-ZM-019.pdf }\end{array}$ \\
\hline 20 & 59122 & $\begin{array}{l}F \\
R \\
P\end{array}$ & $\begin{array}{l}\text { GGGATAAGCAAGTAAAAGCGCTC } \\
\text { CCTTAATTCTCCGCTCATGATCAG } \\
\text { FAM-TTTAAACTGAAGGCG- } \\
\text { GGAAACGACAA-BHQ1 }\end{array}$ & $\begin{array}{l}250 \\
250 \\
200\end{array}$ & 86 & $\begin{array}{l}\text { hitp://gmo-crl.jrc.ec.europa.eu/ } \\
\text { gmomethods/docs/QT-EVE-ZM-012.pdf }\end{array}$ \\
\hline 21 & $B+176$ & $\begin{array}{l}F \\
R \\
P\end{array}$ & $\begin{array}{l}\text { GGCCGTGAACGAGCTGT } \\
\text { GGGAAGAAGCCTACATGTTTTCTAA } \\
\text { FAM-AGCAACCAGATCG- } \\
\text { GCCGACACC-BHQl }\end{array}$ & $\begin{array}{l}300 \\
600 \\
200\end{array}$ & 82 & $\begin{array}{l}\text { hitp://gmo-crl.jrc.ec.europa.eu/ } \\
\text { gmomethods/docs/QT-EVE-ZM-023.pdf }\end{array}$ \\
\hline 22 & $B+11$ & $\begin{array}{l}F \\
R \\
P\end{array}$ & $\begin{array}{l}\text { GCGGAACCCCTATTTGTTA } \\
\text { TCCAAGAATCCCTCCATGAG } \\
\text { FAM-AAATACATTCAAATA- } \\
\text { TGTATCCGCTCA-BHQ1 }\end{array}$ & $\begin{array}{l}750 \\
750 \\
250\end{array}$ & 70 & $\begin{array}{l}\text { http://gmo-crl.jrc.ec.europa.eu/ } \\
\text { gmomethods/docs/QT-EVE-ZM-006.pdf }\end{array}$ \\
\hline 23 & GA21 & $\begin{array}{l}F \\
R \\
P\end{array}$ & $\begin{array}{l}\text { CTTATCGTTATGCTATTTGCAACTTTAGA } \\
\text { TGGCTCGCGATCCTCCT } \\
\text { FAM-CATATACTAACTCATA- } \\
\text { TCTCTTTCTCAACAGCAGGTGGGT- } \\
\text { BHQ1 }\end{array}$ & $\begin{array}{r}150 \\
150 \\
50\end{array}$ & 112 & $\begin{array}{l}\text { hitp://gmo-crl.jrc.ec.europa.eu/ } \\
\text { gmomethods/docs/QT-EVE-ZM-007.pdf }\end{array}$ \\
\hline 24 & MIR 162 & $\begin{array}{l}F \\
R \\
P\end{array}$ & $\begin{array}{l}\text { GCGCGGTGTCATCTATGTTACTAG } \\
\text { TGCCTTATCTGTTGCCTTCAGA } \\
\text { FAM-TCTAGACAATCAGTACATTAAAA- } \\
\text { ACGTCCGCCA-BHQ1 }\end{array}$ & $\begin{array}{l}300 \\
300 \\
150\end{array}$ & 92 & $\begin{array}{l}\text { http://gmo-crl.jrc.ec.europa.eu/ } \\
\text { gmomethods/docs/QT-EVE-ZM-022.pdf }\end{array}$ \\
\hline 25 & MIR604 & $\begin{array}{l}F \\
R \\
P\end{array}$ & $\begin{array}{l}\text { GCGCACGCAATTCAACAG } \\
\text { GGTCATAACGTGACTCCCTTAATTCT } \\
\text { FAM-AGGCGGGAAACGAC- } \\
\text { AATCTGATCATG-BHQ1 }\end{array}$ & $\begin{array}{l}600 \\
300 \\
200\end{array}$ & 76 & $\begin{array}{l}\text { hitp://gmo-crl.jrc.ec.europa.eu/ } \\
\text { gmomethods/docs/QT-EVE-ZM-013.pdf }\end{array}$ \\
\hline 26 & MON810 & $\begin{array}{l}F \\
R \\
P\end{array}$ & $\begin{array}{l}\text { TCGAAGGACGAAGGACTCTAACGT } \\
\text { GCCACCTCCTTTCCACTATCTT } \\
\text { FAM-AACATCCTTTGCCATTG- } \\
\text { CCCAGC-BHQ1 }\end{array}$ & $\begin{array}{l}300 \\
300 \\
180\end{array}$ & 92 & $\begin{array}{l}\text { hitp://gmo-crl.jrc.ec.europa.eu/ } \\
\text { gmomethods/docs/QT-EVE-ZM-020.pdf }\end{array}$ \\
\hline 27 & MON863 & $\begin{array}{l}F \\
R \\
P\end{array}$ & $\begin{array}{l}\text { GTAGGATCGGAAAGCTTGGTAC } \\
\text { TGTTACGGCCTAAATGCTGAACT } \\
\text { FAM-TGAACACCCATCCGAACA- } \\
\text { AGTAGGGTCA-BHQI }\end{array}$ & $\begin{array}{r}150 \\
150 \\
50\end{array}$ & 84 & $\begin{array}{l}\text { hitp://gmo-crl.jrc.ec.europa.eu/ } \\
\text { gmomethods/docs/QT-EVE-ZM-009.pdf }\end{array}$ \\
\hline 28 & MON88017 & $\begin{array}{l}F \\
R \\
P\end{array}$ & $\begin{array}{l}\text { GAGCAGGACCTGCAGAAGCT } \\
\text { TCCGGAGTTGACCATCCA } \\
\text { FAM-TCCCGCCTTCAGTTTAAACA- } \\
\text { GAGTCGGGT-BHQ1 }\end{array}$ & $\begin{array}{r}150 \\
150 \\
50\end{array}$ & 95 & $\begin{array}{l}\text { hitp://gmo-crl.jrc.ec.europa.eu/ } \\
\text { gmomethods/docs/QT-EVE-ZM-016.pdf }\end{array}$ \\
\hline 29 & MON89034 & $\begin{array}{l}F \\
R \\
P\end{array}$ & $\begin{array}{l}\text { TTCTCCATATGACCATCATACTCATT } \\
\text { CGGTATCTATAATACCGTGGTTTTAAA } \\
\text { FAM-ATCCCCGGAAATTATGTT-MGBNFQ }\end{array}$ & $\begin{array}{l}450 \\
450 \\
100\end{array}$ & 77 & $\begin{array}{l}\text { hitp://gmo-crl.jrc.ec.europa.eu/ } \\
\text { gmomethods/docs/QT-EVE-ZM-018.pdf }\end{array}$ \\
\hline 30 & NK603 & $\begin{array}{l}F \\
R \\
P\end{array}$ & $\begin{array}{l}\text { ATGAATGACCTCGAGTAAGCTGTTAA } \\
\text { AAGAGATAACAGGATCCACTCA- } \\
\text { AACACT } \\
\text { FAM-TGGTACCACGCGACACACTTCCA- } \\
\text { CTC-BHQ1 }\end{array}$ & $\begin{array}{r}150 \\
150 \\
50\end{array}$ & 108 & $\begin{array}{l}\text { hitp://gmo-crl.jrc.ec.europa.eu/ } \\
\text { gmomethods/docs/QT-EVE-ZM-008.pdf }\end{array}$ \\
\hline 31 & T25 & $\begin{array}{l}F \\
R \\
P\end{array}$ & $\begin{array}{l}\text { ACAAGCGTGTCGTGCTCCAC } \\
\text { GACATGATACTCCTICCACCG } \\
\text { FAM-TCATTGAGTCGTTCCG- } \\
\text { CCATTGTCG-BHQ1 }\end{array}$ & $\begin{array}{l}400 \\
400 \\
200\end{array}$ & 102 & $\begin{array}{l}\text { hitp://gmo-crl.jrc.ec.europa.eu/ } \\
\text { gmomethods/docs/QT-EVE-ZM-01 1.pdf }\end{array}$ \\
\hline 32 & TC1507 & $\begin{array}{l}F \\
R \\
P\end{array}$ & $\begin{array}{l}\text { TAGTCTTCGGCCAGAATGG } \\
\text { CTTTGCCAAGATCAAGCG } \\
\text { FAM-TAACTCAAGGCCCTCACTCCG- } \\
\text { BHQ1 }\end{array}$ & $\begin{array}{l}300 \\
300 \\
150\end{array}$ & 58 & $\begin{array}{l}\text { hitp://gmo-crl.jrc.ec.europa.eu/ } \\
\text { gmomethods/docs/QT-EVE-ZM-010.pdf }\end{array}$ \\
\hline 33 & MS1 & $\begin{array}{l}F \\
R \\
P\end{array}$ & $\begin{array}{l}\text { ACGCTGCGGACATCTACATT } \\
\text { CTAGATCGGAAGCTGAAGATGG } \\
\text { FAM-CTCATTGCTGATCCACCTA- } \\
\text { GCCGACTT-BHQ1 }\end{array}$ & $\begin{array}{l}400 \\
400 \\
200\end{array}$ & 187 & $\begin{array}{l}\text { http://gmo-crl.jrc.ec.europa.eu/ } \\
\text { gmomethods/docs/QT-EVE-BN-005.pdf }\end{array}$ \\
\hline
\end{tabular}


Table 2 | Continued

\begin{tabular}{|c|c|c|c|c|c|c|}
\hline & Assay & Orientation & Sequences $\left(5^{\prime}-3^{\prime}\right)$ & $\begin{array}{c}\text { Final } \\
\text { concentration }\end{array}$ & $\begin{array}{l}\text { Amplicon } \\
\text { size (bp) }\end{array}$ & Reference \\
\hline 34 & Topas 19/2 & $\begin{array}{l}F \\
R \\
P\end{array}$ & $\begin{array}{l}\text { GTTGCGGTTCTGTCAGTTCC } \\
\text { AGTTCCAAACGTAAAACGGCTT } \\
\text { FAM-TCCCGGTCATATAT- } \\
\text { CAGCGCCGGTC-BHQ1 }\end{array}$ & $\begin{array}{l}400 \\
400 \\
200\end{array}$ & 95 & $\begin{array}{l}\text { http://gmo-crl.jrc.ec.europa.eu/ } \\
\text { gmomethods/docs/QT-EVE-BN-008.pdf }\end{array}$ \\
\hline 35 & OXY-235 & $\begin{array}{l}F \\
R \\
P\end{array}$ & $\begin{array}{l}\text { AGAGAATCGTGAAATTATCTCTACCG } \\
\text { ATTGACCATCATACTCATTGCTGA } \\
\text { FAM-CCATGTAGATTTCCCG- } \\
\text { GACATGAAGCC-BHQ1 }\end{array}$ & $\begin{array}{l}300 \\
300 \\
150\end{array}$ & 105 & Wu G. et al, $2008^{43}$ \\
\hline 36 & MS8 & $\begin{array}{l}F \\
R \\
P\end{array}$ & $\begin{array}{l}\text { GTTAGAAAAAGTAAACAA- } \\
\text { TTAATATAGCCGG } \\
\text { GGAGGGTGTTTTTGGTTATC } \\
\text { FAM-AATATAATCGACGGAT- } \\
\text { CCCCGGGAATTC-BHQ1 }\end{array}$ & $\begin{array}{l}400 \\
400 \\
200\end{array}$ & 130 & $\begin{array}{l}\text { http://gmo-crl.jrc.ec.europa.eu/ } \\
\text { gmomethods/docs/QT-EVE-BN-002.pdf }\end{array}$ \\
\hline 37 & RF3 & $\begin{array}{l}F \\
R \\
P\end{array}$ & $\begin{array}{l}\text { AGCATTTAGCATGTACCATCAGACA } \\
\text { CATAAAGGAAGATGGAGACTTGAG } \\
\text { FAM-CGCACGCTTATCGAC- } \\
\text { CATAAGCCCA-BHQI }\end{array}$ & $\begin{array}{l}400 \\
400 \\
200\end{array}$ & 139 & $\begin{array}{l}\text { http://gmo-crl.jrc.ec.europa.eu/ } \\
\text { gmomethods/docs/QT-EVE-BN-003.pdf }\end{array}$ \\
\hline 39 & T45 & $\begin{array}{l}F \\
R \\
P\end{array}$ & $\begin{array}{l}\text { CAATGGACACATGAATTATGC } \\
\text { GACTCTGTATGAACTGTTCGC } \\
\text { FAM-TAGAGGACCTAA- } \\
\text { CAGAACTCGCCGT-BHQ1 }\end{array}$ & $\begin{array}{l}400 \\
400 \\
200\end{array}$ & 123 & $\begin{array}{l}\text { http://gmo-crl.jrc.ec.europa.eu/ } \\
\text { gmomethods/docs/QT-EVE-BN-001.pdf }\end{array}$ \\
\hline 40 & TT51-1 & $\begin{array}{l}F \\
R \\
P\end{array}$ & $\begin{array}{l}\text { GCGTCCAGAAGGAAAAGGAATA } \\
\text { AGAGACTGGTGATTTCAGCGGG } \\
\text { FAM-ATCTGCCCCAGCACTCGTCCG- } \\
\text { BHQ1 }\end{array}$ & $\begin{array}{l}800 \\
800 \\
400\end{array}$ & 120 & Wu et al, $2013^{44}$ \\
\hline 41 & GTS40-3-2 & $\begin{array}{l}F \\
R \\
P\end{array}$ & $\begin{array}{l}\text { TTCATTCAAAATAAGATC- } \\
\text { ATACATACAGGTT } \\
\text { GGCATTTGTAGGAGCCACCTT } \\
\text { FAM-CCTTTTCCATTTGGG-MGBNFQ }\end{array}$ & $\begin{array}{r}150 \\
150 \\
50\end{array}$ & 84 & $\begin{array}{l}\text { http://gmo-crl.jrc.ec.europa.eu/ } \\
\text { gmomethods/docs/QT-EVE-GM-005.pdf }\end{array}$ \\
\hline 44 & DP-356043 & $\begin{array}{l}F \\
R \\
P\end{array}$ & $\begin{array}{l}\text { GTCGAATAGGCTAGGTTTACGAAAAA } \\
\text { TTTGATATTCTTGGAGTAGACGAGAGTGT } \\
\text { FAM-CTCTAGAGATCCGTCAACATGGTG- } \\
\text { GAGCAC-BHQ1 }\end{array}$ & $\begin{array}{l}750 \\
750 \\
200\end{array}$ & 99 & $\begin{array}{l}\text { http://gmo-crl.jrc.ec.europa.eu/ } \\
\text { gmomethods/docs/QT-EVE-GM-009.pdf }\end{array}$ \\
\hline 45 & A5547-127 & $\begin{array}{l}F \\
R \\
P\end{array}$ & $\begin{array}{l}\text { GCTATTTGGTGGCATTTTTCCA } \\
\text { CACTGCGGCCAACTTACTTCT } \\
\text { FAM-CCGCAATGTCATACC- } \\
\text { GTCATCGTTGTHQ1 }\end{array}$ & $\begin{array}{l}400 \\
400 \\
200\end{array}$ & 75 & $\begin{array}{l}\text { http://gmo-crl.jrc.ec.europa.eu/ } \\
\text { gmomethods/docs/QT-EVE-GM-007.pdf }\end{array}$ \\
\hline 46 & CV-127 & $\begin{array}{l}F \\
R \\
P\end{array}$ & $\begin{array}{l}\text { AACAGAAGTTTCCGTTGAGCTTTAAGAC } \\
\text { CATTCGTAGCTCGGATCGTGTAC } \\
\text { FAM-TTTGGGGAAGCTGTCCCATGCCC- } \\
\text { BHQ1 }\end{array}$ & $\begin{array}{l}400 \\
400 \\
100\end{array}$ & 88 & $\begin{array}{l}\text { http://gmo-crl.jrc.ec.europa.eu/ } \\
\text { gmomethods/docs/QT-EVE-GM-0 1 1.pdf }\end{array}$ \\
\hline 47 & DP-305423 & $\begin{array}{l}F \\
R \\
P\end{array}$ & $\begin{array}{l}\text { CGTGTTCTCTTTTTGGCTAGC } \\
\text { GTGACCAATGAATACATAACACAAACTA } \\
\text { FAM-TGACACAAATGATTTCATAC- } \\
\text { AAAAGTCGAGA-BHQ1 }\end{array}$ & $\begin{array}{l}800 \\
500 \\
220\end{array}$ & 93 & $\begin{array}{l}\text { http://gmo-crl.jrc.ec.europa.eu/ } \\
\text { gmomethods/docs/QT-EVE-GM-008.pdf }\end{array}$ \\
\hline 48 & MON87701 & $\begin{array}{l}F \\
R \\
P\end{array}$ & $\begin{array}{l}\text { TGGTGATATGAAGATACATGCTTAGCAT' } \\
\text { CGTTTCCCGCCTTCAGTTTAAA } \\
\text { TCAGTGTTTGACACACACA- } \\
\text { CTAAGCGTGCC }\end{array}$ & $\begin{array}{l}600 \\
600 \\
250\end{array}$ & 89 & $\begin{array}{l}\text { http://gmo-crl.jrc.ec.europa.eu/ } \\
\text { gmomethods/docs/QT-EVE-GM-010.pdf }\end{array}$ \\
\hline
\end{tabular}


Topas 19/2 and OXY-235 generated the expected results with true positive and negative signals, respectively. The $0.05 \mathrm{ng}$ DNA of both Topas 19/2 and OXY-235 showed near-perfect results with four false negatives for Topas 19/2 and one false positive for OXY-235. For the $0.025 \mathrm{ng}$ and $0.005 \mathrm{ng}$ samples, the number of chambers with a false negative signal increased as the GMO content decreased. The Fluidigm run results suggested that the $0.05 \mathrm{ng}$ of GMO DNA can be consistently detected on a dynamic chip after preamplification, corresponding to 44 copies of the targets. The preamplification proved to be a practical method to increase the size of the template when analyzing real samples. The variations in $\mathrm{Ct}$ values were relatively large among parallels. This result suggested that the distribution of template was not uniform among chambers, resulting in different signal intensities.

Validation of high-throughput PCR on dynamic chips. The Fluidigm 48.48 real-time PCR run was performed to simultaneously detect 48 targets in 48 preamplified samples. Table 2 shows information on the 48 targets. The 48 samples included an NTC control and a blank control, which allowed us to assess the reliability of the dynamic chip runs; 35 single-event samples corresponding to 35 transgenic events at $0.05 \mathrm{ng} / \mu \mathrm{L}$ concentration, and $11 \mathrm{DNA}$ mixtures simulating gene-stacking and mixed samples in practice. The NTC control and blank control (S22 and S47) did not generate any signals, and the MON87701 assay did not generate a signal because of the absence of a MON87701 event. However, most of the TaqMan assays successfully amplified targets from samples known to contain these targets (Fig. 3A). The tabulated data on the presence/absence of targets in samples (Fig. $3 \mathrm{~B}$ ) shows that 33 single-event samples gave excellent amplification results. Sample S10 (59122) gave a false positive result in the chamber corresponding to the T-NOS assay, and S27 (A2704) gave a false negative result in the chamber corresponding to the P-CaMV35S assay. The five samples that contained no common screening targets [S23 (MON89788), S24 (DP356043), S26 (CV-127), S33 (GHB614), and S48 (DP305423)] were accurately identified by event-specific assays. The sensitivity of dynamic chip assays were further demonstrated to be at least $0.05 \mathrm{ng}$ DNA amount for each GM event, corresponding to 19 copies of haploid genome for maize, 25 copies for cotton, 112 copies for rice, 44 copies for rapeseed and soybean. For the 11 complex multiple-event samples, four samples (S36, S37, S40 and S41) produced perfect results, and the other seven samples (S38, S39, S42-S46) produced near-perfect results with minor incidences of false negatives (Fig. 3). This dynamic chip assay exactly detected 35 different events (regulatory elements, functional genes, and GM events) in the mixed sample S40. The successful detection of GM events in complex samples further indicated that the preamplification procedure efficiently increased the amounts of multiple targets.

\section{Discussion}

The dynamic chip-based method developed in this study allowed the simultaneous detection of 48 targets in 48 samples on a Biomark system. In this assay false positive results were rarely observed, indicating that there was no cross-contamination during the preparation of test samples and TaqMan assay mixtures. In practice, sample repeats should be set to rule out the false positive or negative results. If inconsistent results were observed between the repeats, the traditional real-time PCR assays should be performed as a followup to further confirm the test results. The validation results demonstrated that this method could consistently detect samples with a GM DNA amount as low as $0.05 \mathrm{ng}$, and was capable of detecting a variety of GM ingredients in a complex mixture. The strategy of simultaneous detection of multiple targets integrates the GMO screening and identification steps. Therefore, this method can detect not only authorized GMOs, but also new GM events or unauthorized GMOs. The next goal of this research is to establish a 96.96 dynamic chip-based higher-throughput detection method to simultaneously detect and identify 96 targets in 96 samples. In this analysis, the targets would cover major screening elements, all approved GM events, and published unapproved GM events.

Compared with previously established high-throughput methods, our new method has the advantages of flexible throughput, high efficiency, relatively low cost, intuitive results, and suitability for detecting targets in complex samples. The high-throughput method could be used to efficiently and accurately identify GMOs in samples with greatly increased target and sample throughputs. Therefore, this method will be useful for detecting multiple GM events, allowing compliance with GMO regulations in China and other countries.

\section{Methods}

Plant materials. Transgenic materials and non-transgenic seeds were identified and collected by our own GMO detection laboratories. The materials included 14 transgenic maize lines $(3272,59122$, Bt176, Bt11, GA21, MIR162, MIR604, MON810, MON863, MON88017, MON89034, NK603, T25, and TC1507), 7 transgenic soybean lines (40-3-2, A2704-12, MON89788, DP-356043, A5547-127, CV-127, and DP-305423-1), 7 transgenic rapeseed lines (MS1, Topas 19/2, OXY-235, MS8, RF3, RT73, and T45), 1 transgenic rice line (TT51-1) and 6 transgenic cotton lines (MON531, MON88913, MON1445, MON15985, LLCotton25, and GHB614).

DNA extraction. A DNeasy Plant Mini Kit (QIAGEN, Hilden, Germany) was used to extract and purify genomic DNA from seeds or seed powders. DNA purity was checked with a NanoDrop 2000 spectrophotometer (Thermo, Wilmington, DE, USA). The DNA concentrations in samples were determined using a Qubit ${ }^{\circledR} 2.0$ Fluorometer (Life Technologies, Carlsbad, CA USA) with a Qubit ${ }^{\circledR}$ dsDNA BR Assay Kit (Life Technologies). The purified genomic DNA were adjusted to the same concentration of $50 \mathrm{ng} / \mu \mathrm{L}$ using $1 \times \mathrm{TE}$ buffer $(1 \mathrm{mmol} / \mathrm{L}$ Tris, $0.01 \mathrm{mmol} / \mathrm{L}$ EDTA, $\mathrm{pH} 8.0)$, and were stored at $-20^{\circ} \mathrm{C}$ until use.

Preparation of test samples. The genomic DNA solutions extracted from 35 transgenic lines were diluted to $5.0 \mathrm{ng} / \mu \mathrm{L}, 0.5 \mathrm{ng} / \mu \mathrm{L}$, and $0.05 \mathrm{ng} / \mu \mathrm{L}$ levels using salmon sperm DNA, the total DNA concentration for all samples were kept at $50 \mathrm{ng} /$ $\mu \mathrm{L}$. The DNA solutions at a concentration of $0.05 \mathrm{ng} / \mu \mathrm{L}$ were directly used as test samples and labelled as S1-S21, S23-S35, and S48. The DNA solutions at $0.5 \mathrm{ng} / \mu \mathrm{L}$ concentration were used to prepare mixtures of rapeseed, maize, soybean, and cotton DNA by mixing equal volumes of genomic DNA from each line, and labelled as S36S39. Equal volumes of the DNA solutions at a concentration of $5 \mathrm{ng} / \mu \mathrm{L}$ were mixed together to prepare a complex sample, S40, containing 35 events from five crops, and mixed to prepare six other complex samples containing multiple events (S41-S46) from two GM crops: S41 contained 7 rapeseed events and 14 maize events, S42 contained 7 rapeseed events and 7 soybean events, S43 contained 7 rapeseed events and 6 cotton events, S44 contained 14 maize events and 7 soybean events, S45 contained 14 maize events and 6 cotton events, and S46 contained 7 soybean events and 6 cotton events. Two additional control samples were prepared; S22 with salmon sperm DNA as the no template control (NTC), and S47 with $\mathrm{ddH}_{2} \mathrm{O}$ as the blank control. The single-event DNA solutions at $0.5 \mathrm{ng} / \mu \mathrm{L}$ level were also used to test assay sensitivity.

Primers and probes. The oligonucleotide primers and fluorescent dye-labeled TaqMan probes were designed according to validated or previously reported methods. The sequences of the primers and probes were those provided in the original reports. The $5^{\prime}$ ends of all TaqMan ${ }^{\circledR}$ fluorescent probes were labeled with the fluorescent reporter 6-carboxy-fluorescein (FAM), and the $3^{\prime}$ ends were labeled with the fluorescent quencher Black Hole Quencher 1 (BHQ1) or Minor Groove Binder Non-fluorescent Quencher (MGBNFQ). All primers and fluorescent probes were synthesized by Shanghai Sangon Biological Engineering Technology and Services Co. Ltd. (Shanghai, China). Table 2 shows details of the primers and probes.

Real-time PCR. Each TaqMan assay was performed using a CFX96 Real-Time System (Bio-rad, Hercules, CA, USA) in a final volume of $20 \mu \mathrm{L}$, containing $20 \mathrm{ng}$ genomic DNA, $1 \times$ TaqMan Universal PCR Master Mix (Applied Biosystems, Foster City, CA, USA), and the primer/probe set. The final concentrations of primers and probes were those described in published reports or standards (Table 2). All real-time PCR reactions were carried out using the following program: a pre-digestion step of $50^{\circ} \mathrm{C}$ for $2 \mathrm{~min}$; $95^{\circ} \mathrm{C}$ initial denaturation and UNG deactivation step for $10 \mathrm{~min}$; 50 cycles of $15 \mathrm{~s}$ at $94^{\circ} \mathrm{C}$ (denaturation), and $1 \mathrm{~min}$ at $60^{\circ} \mathrm{C}$ (annealing and extension). Fluorescence signals were monitored and analyzed at the annealing and extension steps during each PCR cycle using CFX Manager Version 1.6 (Bio-Rad).

Preamplification. To enrich the amount of template, preamplification was performed for each test sample using the TaqMan PreAmp Master Mix Kit (Life Technologies) according to the manufacturer's protocol, with minor modifications. We included 48 assays in this system. Each primer pair was first diluted to $20 \mu \mathrm{mol} / \mathrm{L}$ with $1 \times$ TE buffer; then, equal volumes $(10 \mu \mathrm{L})$ of all the primer pairs for the 48 assays were mixed to prepare $480 \mu \mathrm{L}$ of pooled assay mix. The preamplification PCR reaction mixture contained the following reagents: $50 \mathrm{ng}$ total DNA (containing salmon sperm DNA and GMO DNA) as template, $1 \times$ TaqMan ${ }^{\circledR}$ PreAmp Master 


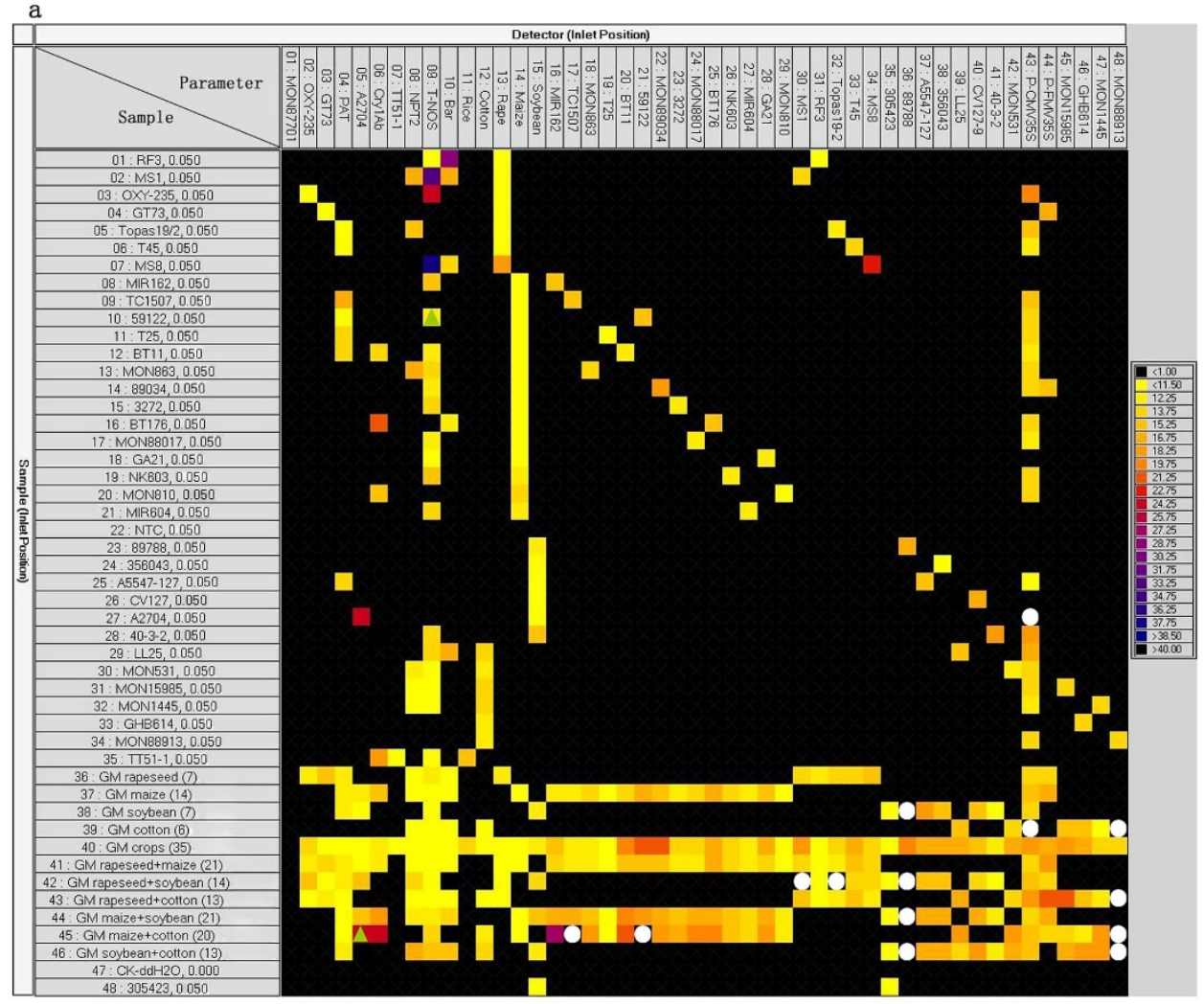

$\mathrm{b}$

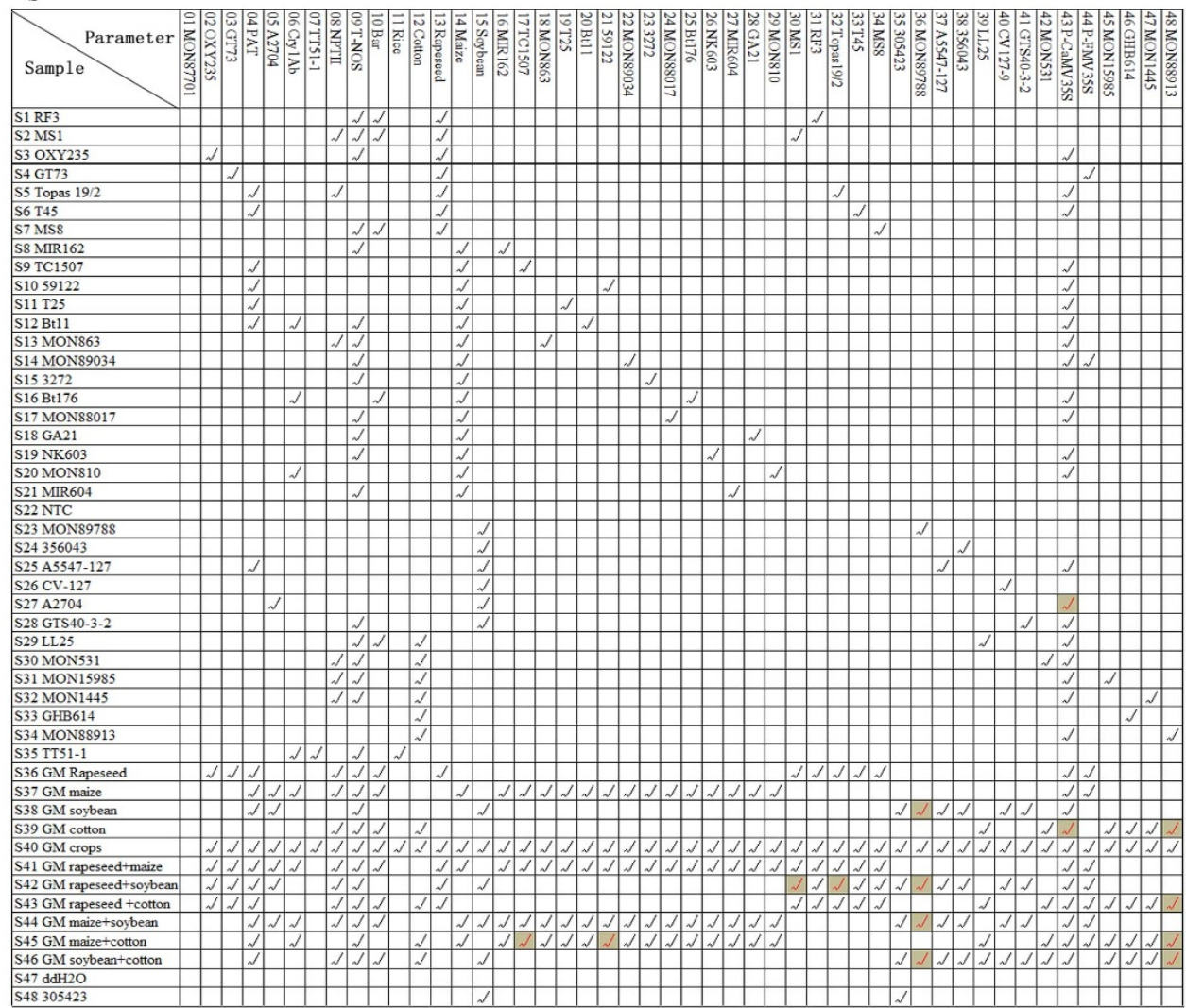

Figure 3 | "Heat map" of 48.48 dynamic array and tabulated data of presence/absence of test targets in samples. (a), Heat map showing TaqMan PCR amplification in a dynamic array chip panel, calculated using Q-PCR Analysis software. Coloured squares indicate positive chambers. Colours on map correspond to Ct values (see colour-coded legend on the right). Green triangles indicate false positive chambers; white circles indicate false negative chambers. (b), Presence of detection targets in the test samples. Tick symbol $(\boldsymbol{V})$ indicates presence of detection targets, grey colour indicates undetected targets in dynamic chip assays. 
Mix, $5 \mu \mathrm{L}$ pooled assay mix, and water to complete the volume to $20 \mu \mathrm{L}$. The preamplifications were carried out on a Bio-Rad C1000 ${ }^{\mathrm{TM}}$ Thermal Cycler (Bio-Rad) using the following protocol from the manufacturer's instructions: initial denaturation at $95^{\circ} \mathrm{C}$ for $10 \mathrm{~min} ; 14$ cycles of $15 \mathrm{~s}$ at $95^{\circ} \mathrm{C}$ (denaturation) and $4 \mathrm{~min}$ at $60^{\circ} \mathrm{C}$ (annealing and extension). Each preamplification product was diluted 20 -fold before use as the template in subsequent PCR reactions.

Real-time PCR on dynamic chips. The Fluidigm 48.48 real-time PCR run was performed according to the manufacturer's instructions (Fluidigm, San Diego, CA, USA). Before performing real-time PCR, the sample mixture and assay mixture were prepared individually. The mixture for each sample (final volume $6 \mu \mathrm{L}$ ) contained $3 \mu \mathrm{L} 2 \times$ TaqMan ${ }^{\circledR}$ Universal PCR Master Mix (Applied Biosystems, PN 4304437), $0.3 \mu \mathrm{L} 20 \times$ GE Sample Loading Reagent (Fluidigm, PN 85000746) and $2.7 \mu \mathrm{L}$ diluted preamplification product as the template. The assay mixture contained $3 \mu \mathrm{L} 2 \times$ Assay Loading Reagent and $3 \mu \mathrm{L} 20 \times$ primer/probe mixture in a final volume of $6 \mu \mathrm{L}$. The final concentrations of primers and probes are shown in Table 2. The samples and assay reagents were loaded into separate reaction chambers on the chip on an IFC controller MX (Fluidigm) after adding $5 \mu \mathrm{L}$ mixture per assay inlet and per sample inlet. The array chip was run on a BioMark HD System (Fluidigm) using a protocol provided in the manufacturer's instructions. The protocol was as follows: a pre-digestion step of $50^{\circ} \mathrm{C}$ for $2 \mathrm{~min} ; 95^{\circ} \mathrm{C}$ initial denaturation and UNG deactivation for $10 \mathrm{~min} ; 50$ cycles of $15 \mathrm{~s}$ at $95^{\circ} \mathrm{C}$ (denaturation) and $1 \mathrm{~min}$ at $60^{\circ} \mathrm{C}$ (annealing and extension). Fluorescence signals were monitored and analyzed at the annealing and extension steps during every PCR cycle using Q-PCR Analysis Software 3.0.2. (Fluidigm).

1. James, C. Global status of commercialized biotech/GM crops: 2013, ISAAA Brief No. 46, http://www.isaaa.org/purchasepublications/itemdescription. asp? ItemType=BRIEFS\&Control=IB046-2013 (2013) Accessed 5 Apr2014.

2. Meyerson, L. A. \& Reaser, J. K. Biosecurity: moving toward a comprehensive approach. BioScience. 52, 593-600 (2002).

3. Marmiroli, N. et al. Methods for detection of GMOs in food and feed. Anal. Bioanal. Chem. 392, 369-384 (2008).

4. Gruere, G. P. \& Rao, S. R. A review of international labeling policies of genetically modified food to evaluate India's proposed rule. Ag. BioForum. 10, 51-64 (2007).

5. Holst-Jensen, A., Rønning, S. B., Lovseth, A. \& Berdal, K. G. PCR technology for screening and quantification of genetically modified organisms (GMOs). Anal. Bioanal. Chem. 375, 985-993 (2003).

6. Querci, M., Van den Bulcke, M., Zel, J., Van den Eede, G. \& Broll, H. New approaches in GMO detection. Anal. Bioanal. Chem. 396, 1991-2002 (2010)

7. Köppel, R., Zimmerli, F. \& Breitenmoser, A. Multiplex real-time PCR for the simultaneous detection and quantification of DNA from three transgenic rice species and construction and application of an artificial oligonucleotide as reference molecule. Eur. Food Res. Technol. 230, 731-736 (2010).

8. Harikai, N. et al. Optional detection of specific genes for genetically modified soybean and maize using multiplex PCR coupled with primer extension on a plastic plate. Biosci. Biotechnol. Biochem. 73, 1886-1889 (2009).

9. Morisset, D., Dobnik, D., Hamels, S., Žel, J. \& Gruden, K. NAIMA: target amplification strategy allowing quantitative on-chip detection of GMOs. Nucleic Acids Res. 36, DOI:10.1093/nar/gkn524|10.1093/nar/gkn524 (2008).

10. Hamels, S. et al. A PCR-microarray method for the screening of genetically modified organisms. Eur. Food Res. Technol. 228, 531-541 (2009).

11. Sua, W., Song, S., Long, M. \& Liu, G. Multiplex polymerase chain reaction/ membrane hybridization assay for detection of genetically modified organisms. J. Biotech. 105, 227-233 (2003).

12. Permingeat, H. R., Reggiardo, M. I. \& Vallejos, R. H. Detection and quantification of transgenes in grains by multiplex and real-time PCR. J. Agric. Food Chem. 50, 4431-4436 (2002).

13. Germini, A. et al. Development of a seven-target multiplex PCR for the simultaneous detection of transgenic soybean and maize in feeds and foods. J. Agric. Food Chem. 52, 3275-3280 (2004).

14. Hernandez, M. et al. Interlaboratory transfer of a PCR multiplex method for simultaneous detection of four genetically modified maize lines: Bt11, MON810, T25, and GA21. J. Agric. Food Chem. 53, 3333-3337 (2005).

15. Leimanis, S. et al. A microarray-based detection system for genetically modified (GM) food ingredients. Plant Mol. Biol. 61, 123-139 (2006).

16. Leimanis, S. et al. Validation of the performance of a GMO multiplex screening assay based on microarray detection. Eur. Food Res. Technol. 227, 1621-1632 (2008).

17. Bordoni, R., Germini, A., Mezzelani, A., Marchelli, R. \& De Bellis, G. A microarray platform for parallel detection of five transgenic events in foods: a combined polymerase chain reaction-ligation detection reaction-universal array method. J. Agric. Food Chem. 53, 912-918 (2005).

18. Nadal, A., Coll, A., La Paz, J. L., Esteve, T. \& Pla, M. A new PCR-CGE (size and color) method for simultaneous detection of genetically modified maize events. Electrophoresis. 27, 3879-3888 (2006).

19. Nadal, A., Esteve, T. \& Pla, M. Multiplex polymerase chain reaction-capillary gel electrophoresis: a promising tool for GMO screening-assay for simultaneous detection of five genetically modified cotton events and species. J. AOAC Int. 92, 765-772 (2009).
20. Rudi, K., Rud, I. \& Holck, A. A novel multiplex quantitative DNA array based PCR (MQDA-PCR) for quantification of transgenic maize in food and feed. Nucl. Acids Res. 31, e62; DOI: 10.1093/nar/gng061 (2003).

21. Xu, J. et al. Event-specific detection of seven genetically modified soybean and maizes using multiplex-PCR coupled with oligonucleotide microarray. J. Agric. Food Chem. 55, 5575-5579 (2007).

22. Markoulatos, P., Siafakas, N. \& Moncany, M. Multiplex polymerase chain reaction: a practical approach. J. Clin. Lab. Anal. 16, 47-51 (2002).

23. Chaouachi, M. et al. A high- throughput multiplex method adapted for GMO detection. J. Agric. Food Chem. 56, 11596-11606 (2008).

24. Guo, J. et al. MPIC: A High-Throughput Analytical Method for Multiple DNA Targets. Anal. Chem. 83, 1579-1586 (2011).

25. Shao, N. et al. MACRO: a combined microchip-pcr and microarray system for high-throughput monitoring of genetically modified organisms. Anal. Chem. 86, 1269-1276 (2014).

26. Querci, M. et al. Real-time PCR-based ready-to-use multi-target analytical system for GMO detection. Food Anal. Methods. 2, 325-336 (2009).

27. Cottenet, G., Blancpain, C., Sonnard, V. \& Chuah, P. F. Development and validation of a multiplex real-time PCR method to simultaneously detect 47 targets for the identification of genetically modified organisms. Anal. Bioanal. Chem. 405, 6831-6844 (2013).

28. Huggett, J. F. et al. The digital MIQE guidelines: minimum information for publication of quantitative digital PCR experiments. Clin. Chem. 59, 6892-6902 (2013).

29. Azuara, D. et al. Nanofluidic Digital PCR for KRAS Mutation Detection and Quantification in Gastrointestinal Cancer. Clin. Chem. 58, 1332-1341 (2012)

30. Wang, J. et al. Quantifying EGFR alterations in the lung cancer genome with nanofluidic digital PCR arrays. Clin. Chem. 56, 4623-4632 (2010).

31. Whale, A. S. et al. Comparison of microfluidic digital PCR and conventional quantitative PCR for measuring copy number variation. Nucleic Acids Res. 40, e82; DOI:10.1093/nar/gks203 (2012).

32. Burns, M. J., Burrell, A. M. \& Foy, C. A. The applicability of digital PCR for the assessment of detection limits in GMO analysis. Eur. Food Res. Technol. 231, 353-362 (2010)

33. Huelga, S. C. et al. Integrative genome-wide analysis reveals cooperative regulation of alternative splicing by hnRNP proteins. Cell Rep. 1, 167-178 (2012).

34. Sanchez-Freire, V., Ebert, A. D., Kalisky, T., Quake, S. R. \& Wu, J. C. Microfluidic single-cell real-time PCR for comparative analysis of gene expression patterns. Nat. Protoc. 7, 829-838 (2012).

35. Spurgeon, S. L., Jones, R. C. \& Ramakrishnan, R. High throughput gene expression measurement with real time PCR in a microfluidic dynamic array. PLoS ONE. 3, e1662; DOI:10.1371/journal.pone.0001662 (2008).

36. Jang, J. S. et al. Quantitative miRNA expression analysis using fluidigm microfluidics dynamic arrays. BMC Genomics. 12, 144; DOI:10.1186/1471-2164-12-144 (2011).

37. Brod, F. C. A. et al. A high-throughput method for GMO multi-detection using a microfluidic dynamic array. Anal. Bioanal. Chem. 406, 1397-1410 (2014).

38. Arumuganathan, K. \& Earle, E. D. Nuclear DNA content of some important plant species. Plant Mol. Biol. Report. 9, 211-215 (1991)

39. Grohmann, L., Brunen-nieweler, C., Nemeth, A. \& Waiblinger, H. Collaborative Trial Validation Studies of Real-Time PCR-Based GMO Screening Methods for Detection of the bar Gene and the ctp2-cp4epsps Construct. J. Agric. Food Chem. 57, 8913-8920 (2009).

40. Zhu, S. et al. Protocol of the real-time PCR for detecting genetically modified plants and their derived products. Entry-exit inspection and quarantine industry standard of the People's Republic of China. SN/T1204-2003, 1-5. http://www. bjstandard.com/standard/166054.html (2003) Date of access: 5 Feb. 2013.

41. Lu, C. et al. Detection of genetically modified plants and derived productsQualitative PCR method for the marker genes NPTII.HPT and PMI. Announcement by the Ministry of Agriculture. No.1782-2-2012, 1-9. http://www. bjstandard.com/standard/271346.html (2012) Date of access: 5 Feb. 2013.

42. Zeitler, R., Pietsch, K. \& Waiblinger, H. U. Validation of real-time PCR methods for the quantification of transgenic contaminations in rapeseed. Eur. Food Res. Technol. 214, 346-351 (2002).

43. Wu, G., Wu, Y., Xiao, L. \& Lu, C. Event-specific qualitative and quantitative PCR methods for the detection of genetically modified rapeseed OXY-235. Transgenic Res. 17, 851-862 (2008)

44. Wu, Y. et al. Collaborative validation of an event-specific quantitative real-time PCR method for genetically modified rice event TT51-1 detection. J. Agric. Food Chem. 61, 5953-5960 (2013).

\section{Acknowledgments}

This study was supported by a grant from the National Major Special Project for the Development of Transgenic Organisms of China (grant no. 2014ZX08012-003 and 2014ZX0801202B), the National Natural Science Foundation of China (grant no. 31271880), and Jilin Agricultural Science and Technology Innovation Program (2013).

\section{Author contributions}

G.W. and F.L. designed this experiment; G.W. and Y.W. wrote the main manuscript; Y.W. and X.L. prepared the figures; X.L. and J.L. performed this experiment; Y.L. and L.L. advised on the experimental design and reviewed the manuscript. 


\section{Additional information}

Competing financial interests: The authors declare no competing financial interests.

(i) This work is licensed under a Creative Commons Attribution 4.0 International License. The images or other third party material in this article are included in the article's Creative Commons license, unless indicated otherwise in the credit line; if

How to cite this article: Li, X. et al. Development and Validation of A 48-Target Analytical Method for High-throughput Monitoring of Genetically Modified Organisms. Sci. Rep. 5, 7616; DOI:10.1038/srep07616 (2015).

the material is not included under the Creative Commons license, users will need to obtain permission from the license holder in order to reproduce the material. To view a copy of this license, visit http://creativecommons.org/licenses/by/4.0/ 